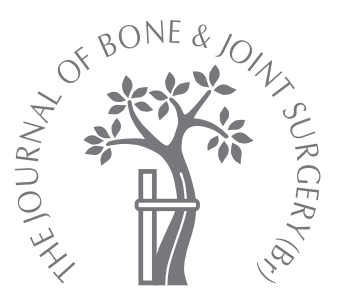
R. Kotnis,
C. Pasapula,
F. Anwar,
P. H. Cooke,
R. J. Sharp

From the Nuffield

Orthopaedic Centre,

Oxford, England

\title{
The management of failed ankle replacement
}

\begin{abstract}
Advances in the design of the components for total ankle replacement have led to a resurgence of interest in this procedure.

Between January 1999 and December 2004, 16 patients with a failed total ankle replacement were referred to our unit. In the presence of infection, a two-stage salvage procedure was planned. The first involved the removal of the components and the insertion of a cement spacer. Definitive treatment options included hindfoot fusion with a circular frame or amputation. When there was no infection, a one-stage salvage procedure was planned. Options included hindfoot fusion with an intramedullary nail or revision total ankle replacement. When there was suspicion of infection, a percutaneous biopsy was performed. The patients were followed up for a minimum of 12 months.
\end{abstract}

Of the 16 patients, 14 had aseptic loosening, five of whom underwent a revision total ankle replacement and nine a hindfoot fusion. Of the two with infection, one underwent fusion and the other a below-knee amputation. There were no cases of wound breakdown, nonunion or malunion.

Management of the failed total ankle replacement should be performed by experienced surgeons and ideally in units where multidisciplinary support is available. Currently, a hindfoot fusion appears to be preferable to a revision total ankle replacement.

Advances in the design of the components of ankle replacements have led to a resurgence of interest in this procedure. This increase in the number of primary procedures is coupled with an increase in revisions. The quoted rate of failure for these newer designs is approximately $3 \%$ per year; and after ten years of implantation at this rate, about 200 revisions would be required every year. ${ }^{1-9}$

The first generation of total ankle replacements (TAR) was beset by problems of early failure, with re-operation rates as high as $41 \% .{ }^{10}$ However, this has significantly decreased after improvements in design. ${ }^{1,6-8,11-13}$

The factors associated with early failure include wound problems, deep infection, poor osseo-integration and mis-sizing and malpositioning of the components. ${ }^{14,15}$

The paucity of revision implants, poor softtissue cover and vascularity and decreased bone stock make revision more challenging than for failed hip or knee arthroplasties. There are very few studies which have addressed the management of failed ankle replacements. ${ }^{16-19}$ We report our experience of managing these difficult cases and suggest an algorithm to guide the treatment of these patients.

\section{Patients and Methods}

Between January 1999 and December 2004, 16 patients with a failed TAR were referred to our tertiary unit. Definitive treatment was performed by one of two foot and ankle surgeons (PHC).

There were eight men and eight women. The mean age of the men was 64.7 years (53 to 74 ) and of the women 60.6 years (51 to 68). Table I shows the diagnosis prior to primary ankle replacement and the implant used. Of the 16 patients, 13 had had their primary TAR performed elsewhere and were referred for further management.

Since 1999, a standard protocol has been used for managing patients with a failed TAR (Fig. 1). Failure is defined as persistent pain with radiological signs of loosening of the implant. Initial investigations including a full blood count, erythrocyte sedimentation rate and level of C-reactive protein and standing plain radiography of the affected ankle (anteroposterior and lateral views) were performed on each patient. Radiological signs of loosening included radiolucent lines around the components, or malposition and subsidence of the component. 
Failed total ankle replacement

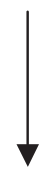

Blood tests and standing plain radiographs

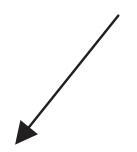

Signs of infection

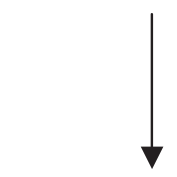

Two-stage revision

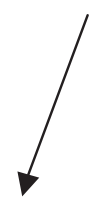

Amputation

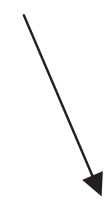

Fusion with Ilizarov frame
Revision total

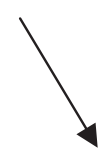

No sign of infection (percutaneous biopsy)

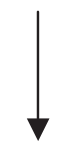

One-stage revision

(Frozen section if suspicious of infection)

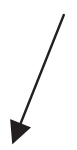
ankle replacement

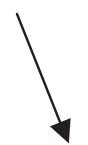

Hindfoot fusion
An implant was considered to be infected when there were local signs of infection and raised inflammatory markers. In patients with an equivocal history and clinical examination or blood indices, a radiologically-guided percutaneous biopsy was performed.

All the patients were reviewed by the specialist bone infection team which looked after the microbiological and the chemotherapeutic aspects of their subsequent management.

Protocol for infected total ankle replacements. When infection was suspected, a two-stage salvage procedure was planned. The first stage involved a thorough debridement with removal of the components and all non-viable soft tissue and insertion of a gentamicin-loaded cement spacer. Multiple tissue samples were obtained and analysed. Appropriate antibiotics were given after sampling according to previous protocols used in our unit for hip and knee arthroplasty. ${ }^{20}$ The patient's response to treatment was assessed by the clinical findings and level of serum inflammatory markers. ${ }^{21,22}$ The timing of the second-stage procedure was decided in conjunction with the bone infection physicians. A minimum duration of six weeks of empirical antibiotic therapy formed a part of our protocol.

The treatment options at the second stage included an ankle fusion using a circular external fixator (Ilizarov frame) or a transtibial amputation. In the presence of a leglength discrepancy of more than $3 \mathrm{~cm}$, simultaneous dis- traction osteogenesis within the circular frame to lengthen the tibia proximally was considered. At surgery, the cement spacer was removed and the distal tibia excised to viable bleeding bone. A proximal tibial corticotomy was performed and fixation achieved using a circular frame spanning the ankle. Distraction was performed at the standard rate of $1 \mathrm{~mm}$ per day.

Amputation was offered as the primary procedure after a decision made by the two senior surgeons (RS, PC). All the patients had extensive pre-operative counselling and a prosthetist was made available to discuss the options available for an appropriate prosthesis.

Protocol for non-infected total ankle replacements. When infection was not suspected, a revision was planned as a one-stage procedure. Treatment was tailored to the individual needs of each patient. The surgical options included a revision TAR or a hindfoot arthrodesis. Revision was performed using an anterior approach. The components were removed and a stemmed tibial component with a standard talar component (Scandinavian TAR (STAR)) was used. Cement was used to fill any large bone defects.

Hindfoot arthrodesis was performed using a locked compression intramedullary nail (Biomet UK Ltd., Bridgend, United Kingdom) with distal fibula as the bone graft.

Factors influencing the type of treatment included the quality of bone, the degree of bone loss, previous ipsilateral 
Table I. Clinical details, cause of failure, previous procedures and the definitive procedure for the 16 patients

\begin{tabular}{|c|c|c|c|c|c|c|c|}
\hline Case & $\begin{array}{l}\text { Age } \\
\text { (yrs) }\end{array}$ & Gender & Diagnosis $^{*}$ & $\begin{array}{l}\text { Primary } \\
\text { prosthesis }^{\dagger}\end{array}$ & Revision procedure before definitive treatment & Cause of failure & $\begin{array}{l}\text { Definitive } \\
\text { procedure (mths) }\end{array}$ \\
\hline 1 & 51 & $\mathrm{~F}$ & PTA & STAR & $\begin{array}{l}\text { Arthroscopy and debridement with revision of the } \\
\text { talar component and fixation of a distal fibular } \\
\text { fracture at } 30 \mathrm{mths}\end{array}$ & Asepic loosening & Arthrodesis (38) \\
\hline 2 & 64 & $\mathrm{~F}$ & PTA & STAR & $\begin{array}{l}\text { Debridement of lateral impingement at } 8 \mathrm{mths} \\
\text { Talar component revision at } 24 \mathrm{mths} \\
\text { Revision of tibial component at } 48 \mathrm{mths}\end{array}$ & Aseptic loosening & Arthrodesis (63) \\
\hline 3 & 60 & $\mathrm{M}$ & $\mathrm{OA}$ & $\mathrm{BP}$ & Revision of all component at 12 mths & Aseptic loosening & Arthrodesis (48) \\
\hline 4 & 53 & $\mathrm{M}$ & RA & STAR & & Aseptic loosening & Arthrodesis (30) \\
\hline 5 & 58 & $\mathrm{~F}$ & RA & STAR & Arthroscopy and synovectomy at $24 \mathrm{mths}$ & Aseptic loosening & Arthrodesis (36) \\
\hline 6 & 60 & $\mathrm{~F}$ & OA & STAR & Heel shift to correct hindfoot alignment at $32 \mathrm{mths}$ & Aseptic loosening & Arthrodesis (43) \\
\hline 7 & 64 & $\mathrm{~F}$ & OA & STAR & Heel shift to correct hindfoot alignment at $28 \mathrm{mths}$ & Aseptic loosening & Arthrodesis (36) \\
\hline 8 & 65 & $\mathrm{M}$ & $\mathrm{OA}$ & STAR & & Aseptic loosening & Arthrodesis (34) \\
\hline 9 & 68 & $\mathrm{~F}$ & OA & STAR & Arthroscopy and synovectomy at $30 \mathrm{mths}$ & Aseptic loosening & Revision \\
\hline 10 & 72 & $\mathrm{M}$ & $\mathrm{OA}$ & STAR & $\begin{array}{l}\text { Arthroscopy and biopsy at } 9 \mathrm{mths} \\
\text { Exchange of loose talar component at } 14 \mathrm{mths}\end{array}$ & Aseptic loosening & Arthrodesis (30) \\
\hline 11 & 68 & $\mathrm{~F}$ & OA & STAR & Revision of meniscal component at $22 \mathrm{mths}$ & Aseptic loosening & Revision \\
\hline 12 & 60 & $\mathrm{M}$ & RA & AGILITY & & Septic loosening & $\begin{array}{l}\text { Below-level } \\
\text { amputation (23) }\end{array}$ \\
\hline 13 & 52 & $\mathrm{~F}$ & PTA & STAR & $\begin{array}{l}\text { Excision of excess anterior bone for impingement at } \\
12 \mathrm{mths}\end{array}$ & Septic loosening & llizarov (25) \\
\hline 14 & 65 & $\mathrm{M}$ & $\mathrm{OA}$ & STAR & & Aseptic loosening & Revision \\
\hline 15 & 69 & M & OA & STAR & & Aseptic loosening & Revision \\
\hline 16 & 74 & $\mathrm{M}$ & OA & STAR & & Aseptic loosening & Revision \\
\hline
\end{tabular}

* PTA, post-traumatic arthritis; OA, osteoarthritis; RA, rheumatoid arthritis

† STAR, Scandinavian total ankle replacement; BP, Buechel-Pappas total ankle replacement

foot and ankle surgical procedures, patient comorbidities and patient choice. Of these a lack of available bone stock was the most important, since it precluded any type of revision.

If there was macroscopic evidence of infection at operation, an intra-operative frozen section was obtained. If positive for infection, the planned procedure was abandoned and the protocol for an infected implant was used.

Post-operative regimen. The operated foot was elevated and placed in a plaster-of-Paris backslab for 24 hours. Thromboembolic prophylaxis with low-molecular-weight fragmin was used until the patient was mobile. All patients were given antibiotics for seven days after surgery and thereafter prolonged therapy was used only in those in whom samples had been suggestive of infection. Fortunately, in this series no patient with revision or fusion had infection.

Patients who underwent a fusion with circular external fixation were allowed to bear weight immediately but those who had fusion with a retrograde intramedullary nail did not bear weight for six weeks and then progressed from partial to full weight-bearing over three months. Patients who had amputation were rehabilitated appropriately and those with a revision were allowed to bear weight in a removable cast as tolerated. All the patients were followed up for a minimum of 12 months.

\section{Results}

None of the patients was lost to follow-up.

All had pre-operative radiological evidence of loosening or subsidence of the component. On the tibial side, there were both radiolucent lines and subsidence, but on the talar side only subsidence.

Two patients presented with deep infection. In one of these the infection was presumed to be secondary to immunosuppressive therapy for rheumatoid arthritis. The time to presentation from the TAR was 25 months and 23 months, respectively. In both patients, there was evidence of local infection and the level of serum inflammatory markers was raised. Deep tissue intra-operative specimens confirmed the diagnosis with Gram-positive cocci present in both patients. In addition, diphtheroids were cultured from one patient. The ankle replacements were removed and empirical antibiotic therapy began.

A total of 14 patients presented with persistent pain and swelling secondary to aseptic loosening. Of these four had previously undergone a revision of one or both components (Table I). Analysis of the immediate postoperative radiographs obtained after primary surgery showed that the implant was oversized in four patients and there was a degree of malalignment in five. In addition, two of these five patients had an oversized component.

\section{Definitive treatment}

Septic loosening. Both the patients with infection underwent treatment according to the protocol described earlier. Hindfoot fusion using an Ilizarov frame was performed in one (Fig. 2). The loss of limb length of $5 \mathrm{~cm}$ on the affected side was treated by distraction osteogenesis using proximal fibular and tibial corticotomies. Radiological fusion was achieved at four months and the frame was removed at five months. 


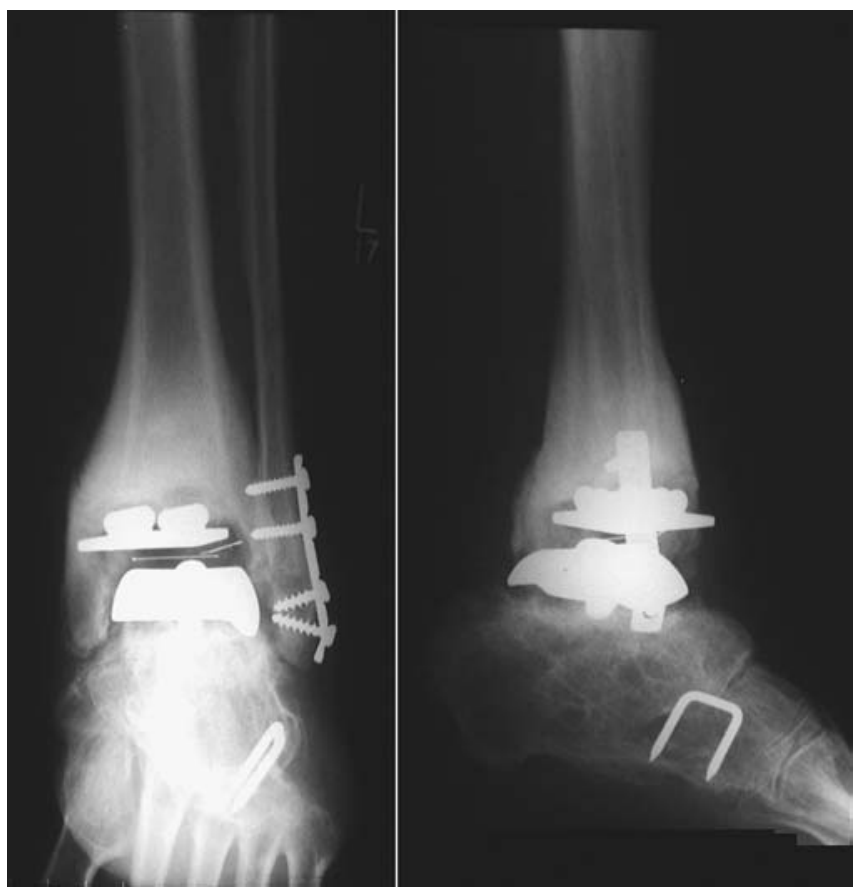

Fig. 2a

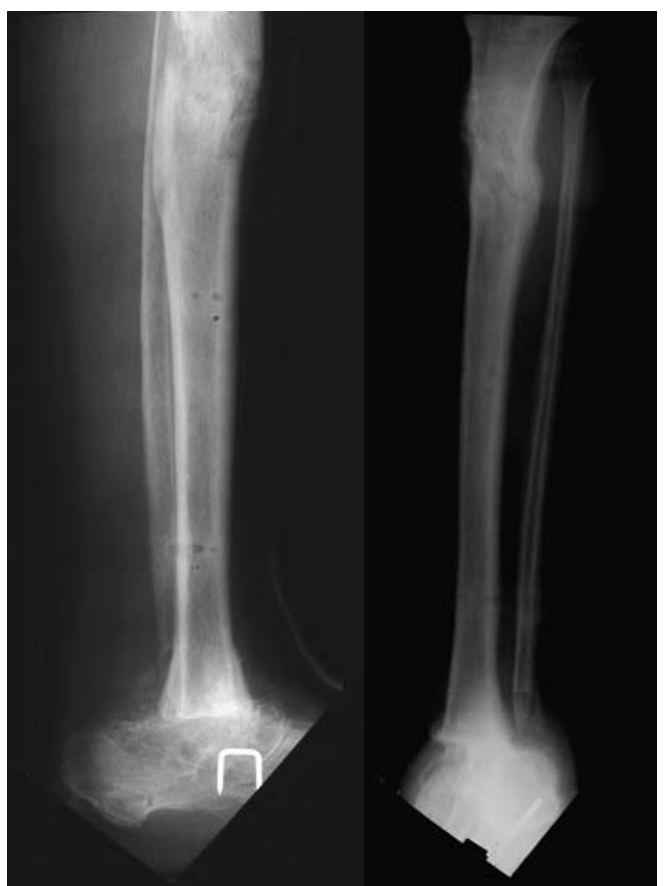

Fig. 2c

The second patient, with an Agility TAR (DePuy Orthopaedics, Leeds, United Kingdom), had evidence of extensive bone loss on plain radiography and an ongoing infection despite intravenous antibiotic therapy. A below-knee amputation was advised and performed in view of the patient's age, medical co-morbidities and personal experience of relatives with Ilizarov frames (Fig. 3). fusion.

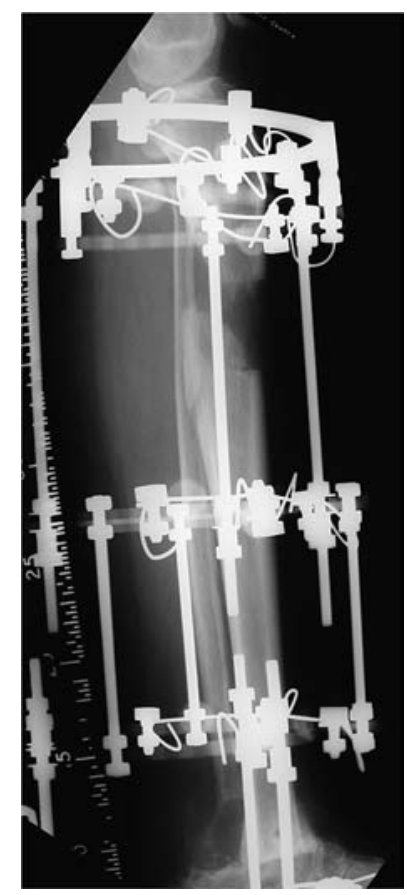

Fig. $2 b$

Radiographs of a patient with an infected and loose Scandinavian total ankle replacement showing a) fixation of a previous implant-related distal fibular fracture, b) management by hindfoot fusion and distraction osteogenesis in an llizarov frame and c) healed proximal corticotomy and union of the hindfoot

Aseptic loosening. Five patients underwent revision with a STAR (Fig. 4). Revision was performed at 4, 20, 24, 30 and 48 months respectively, after the primary procedure. One patient with a Buechel-Pappas (Endotec Orthopaedics, South Orange, New Jersey) TAR had undergone a revision at 12 months and was revised to hindfoot fusion (Fig. 5). 


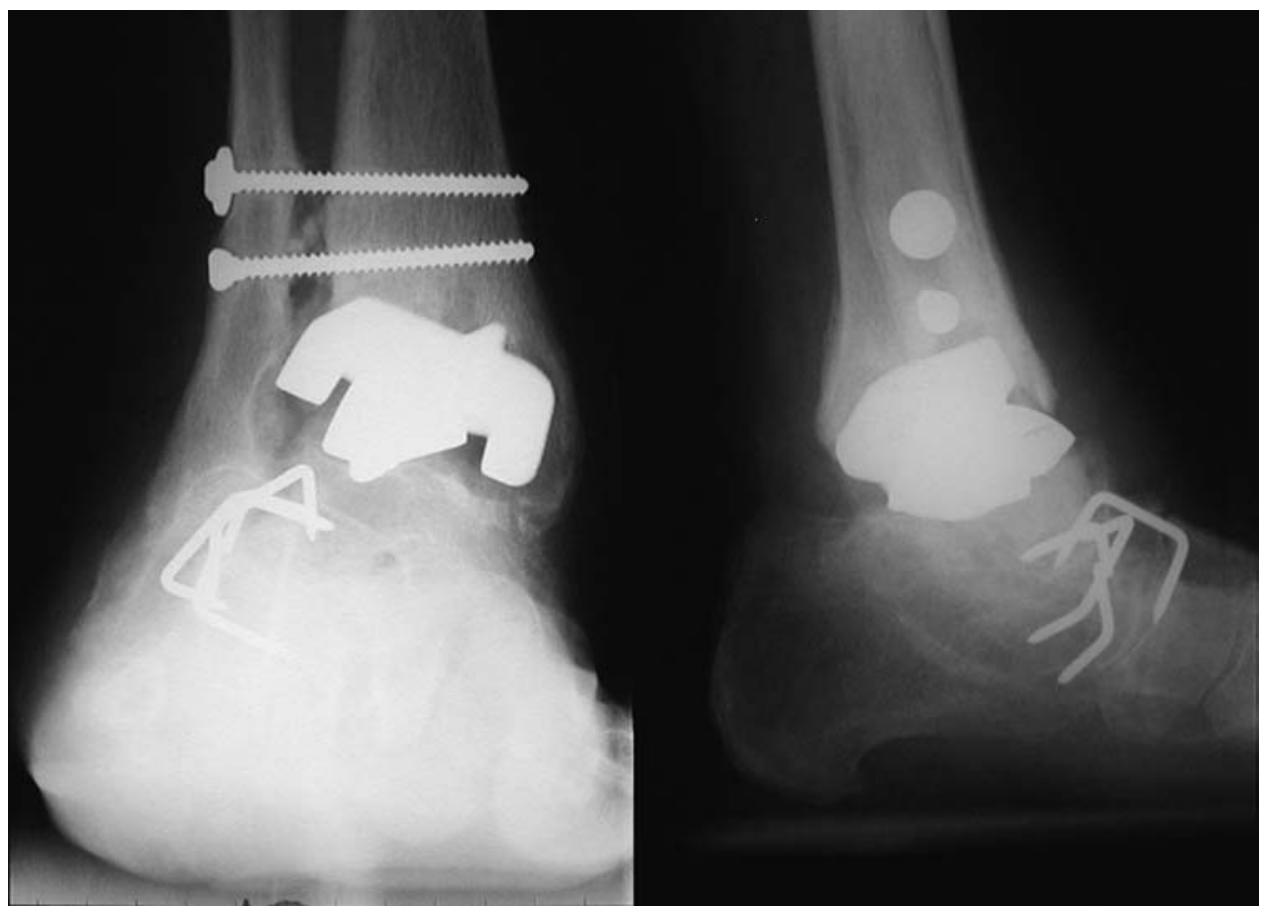

Fig. 3a

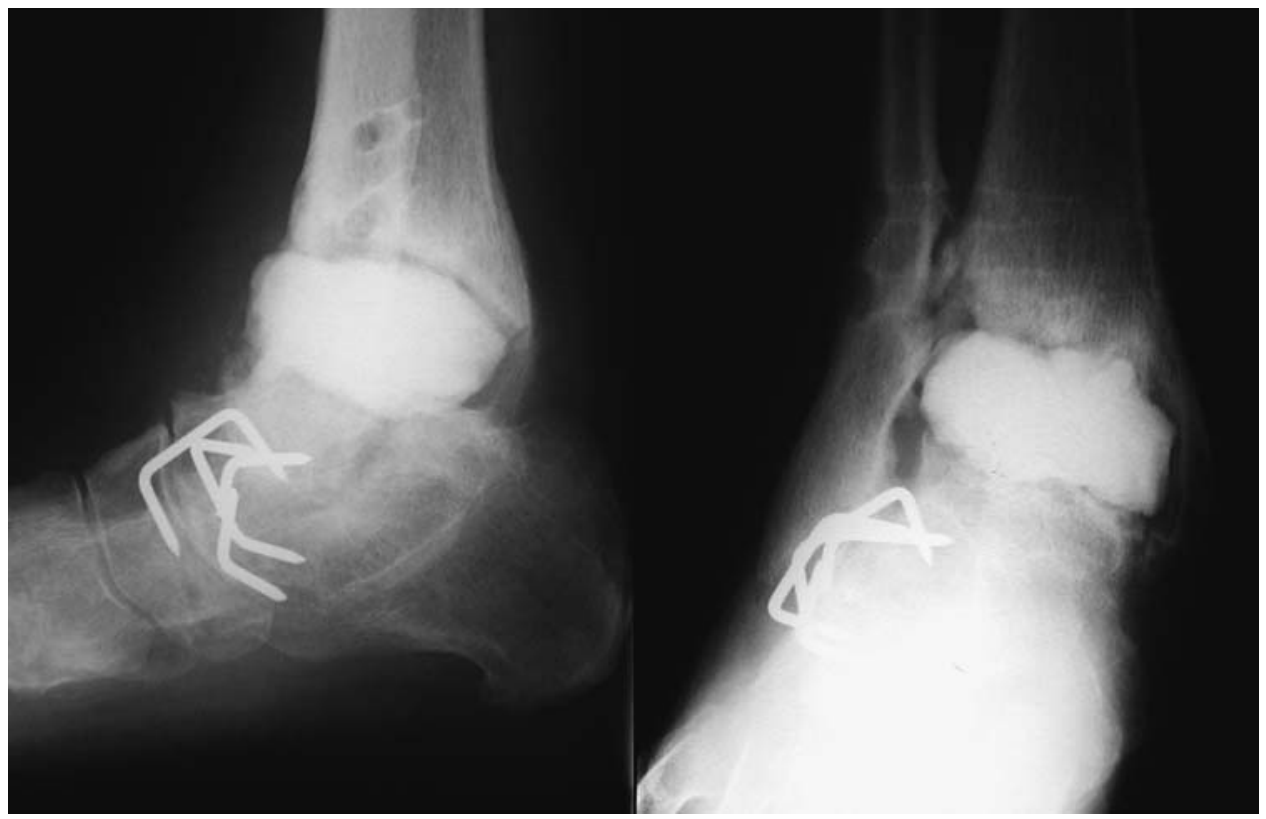

Fig. 3b

Radiographs of a patient with an infected and loose Agility total ankle replacement showing a) evidence of loosening of both components, and b) removal of the components and insertion of a cement spacer. A belowknee amputation was performed.

In nine patients, the hindfoot fusion was performed with a locked intramedullary retrograde nail (Biomet, Warsaw, Indiana) (Fig. 5). Four of these patients had previously undergone revision of one or both components. Clinical evidence of fusion was achieved at a mean of 3.5 months (2.5 to 4.5$)$ and radiological fusion by 8 months ( 5 to 10$)$.

There were no cases of wound breakdown, nonunion or malunion at follow-up at 12 months although the radiological assessment of union under these circumstances is difficult. 


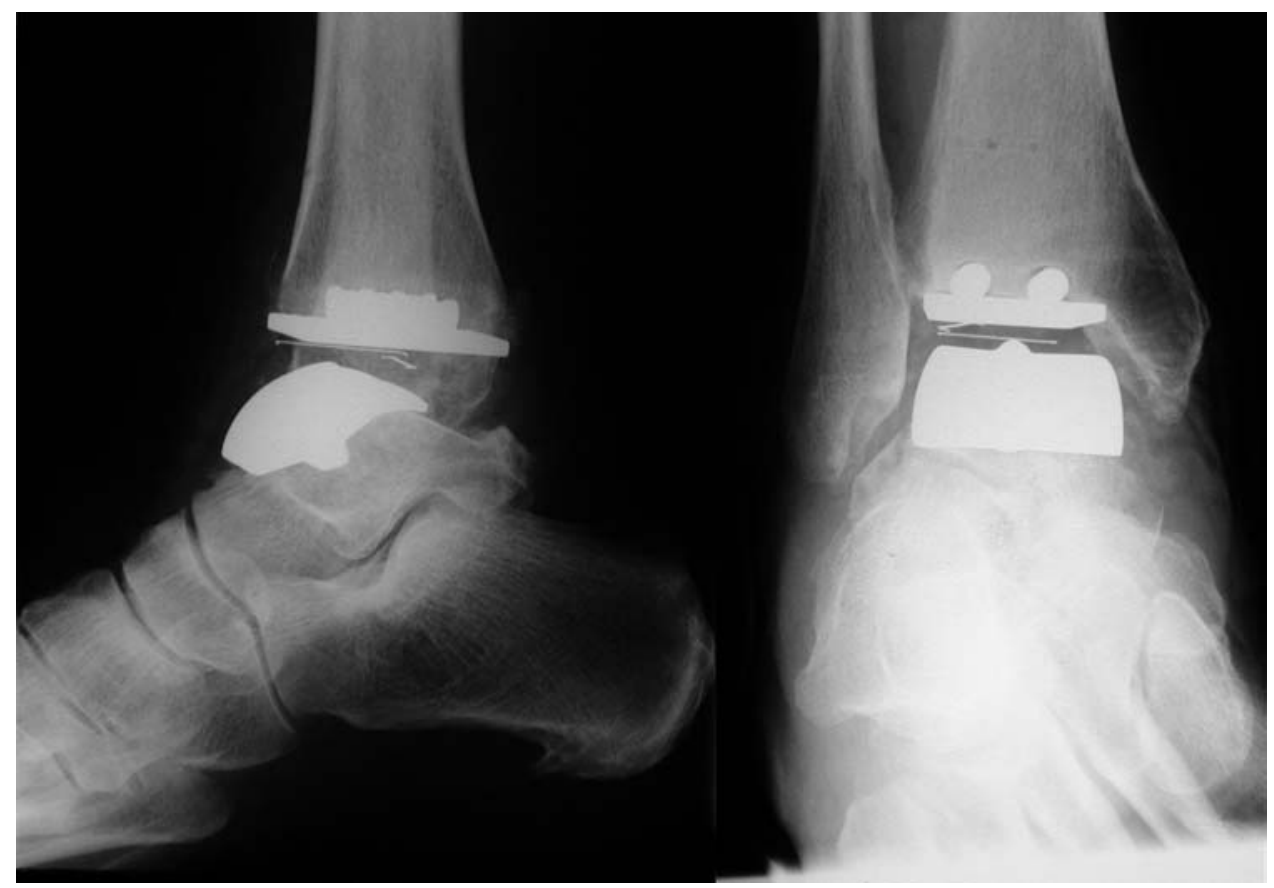

Fig. $4 a$

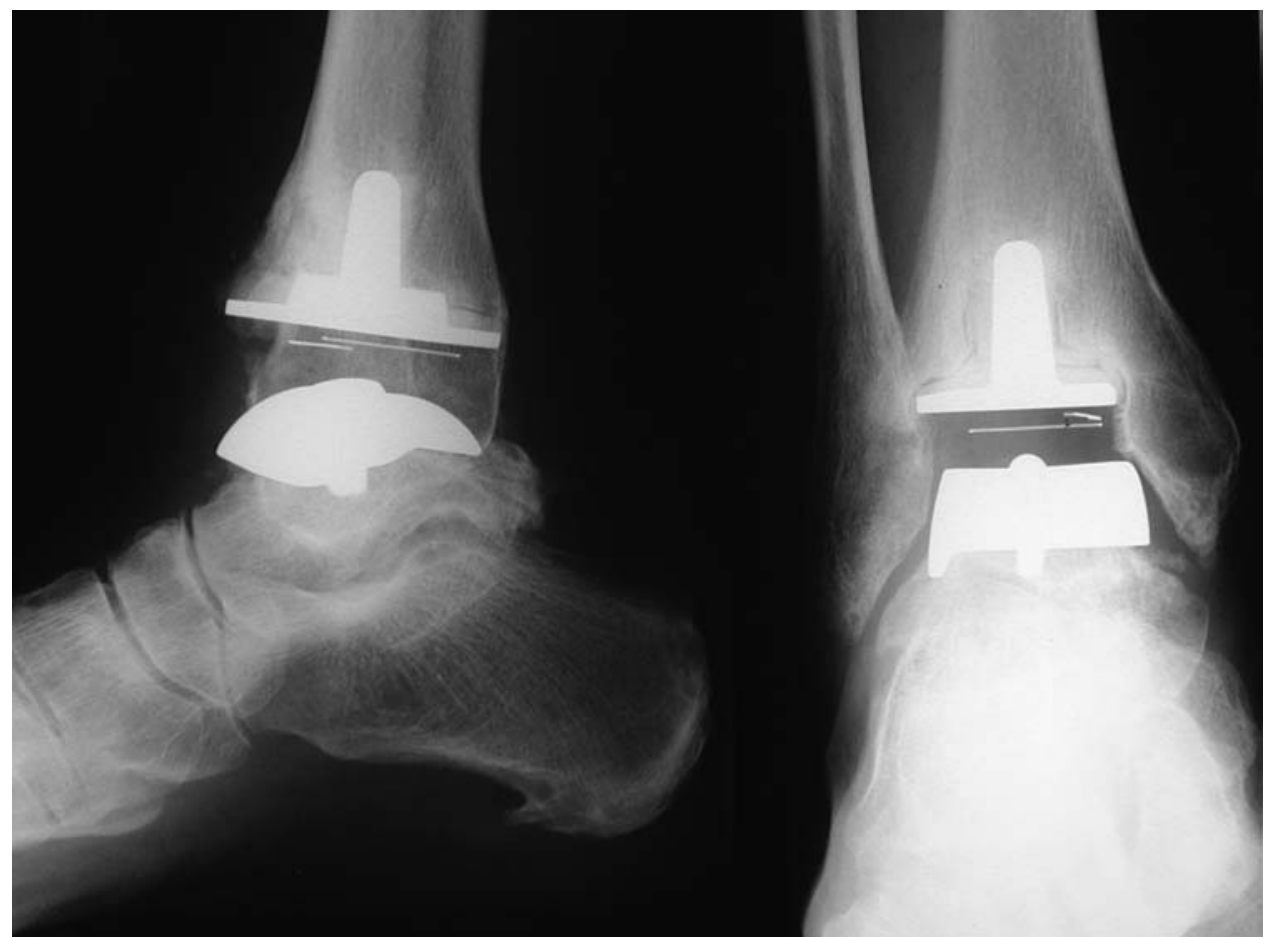

Fig. $4 b$

Radiographs showing a) aseptic loosening of a Scandinavian total ankle replacement (STAR) and b) revision using a stemmed revision STAR tibial component and a STAR talar component with improved alignment.

Complications. Of the ten patients who underwent a fusion, two complained of persistent pain in the ankle. No orthopaedic cause could be identified and the patients were referred to the pain clinic for further management. Two patients with a revision TAR still had pain on weight-bearing at follow-up at 12 months. In one of these, the prosthesis showed radiological signs of failure but the patient declined further surgery. 


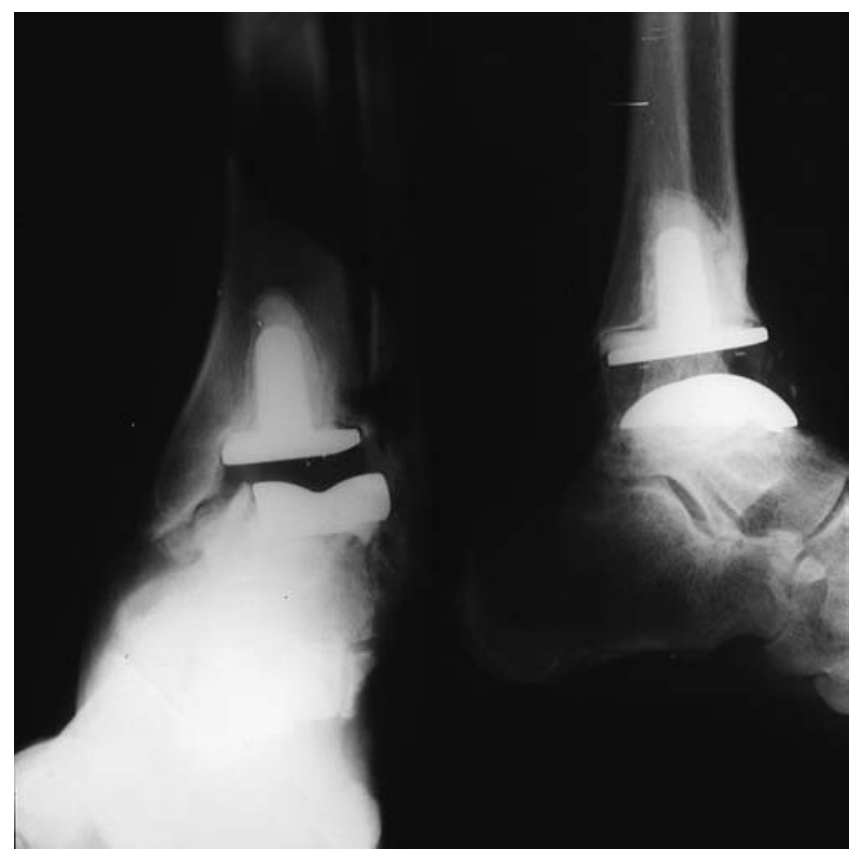

Fig. $5 a$

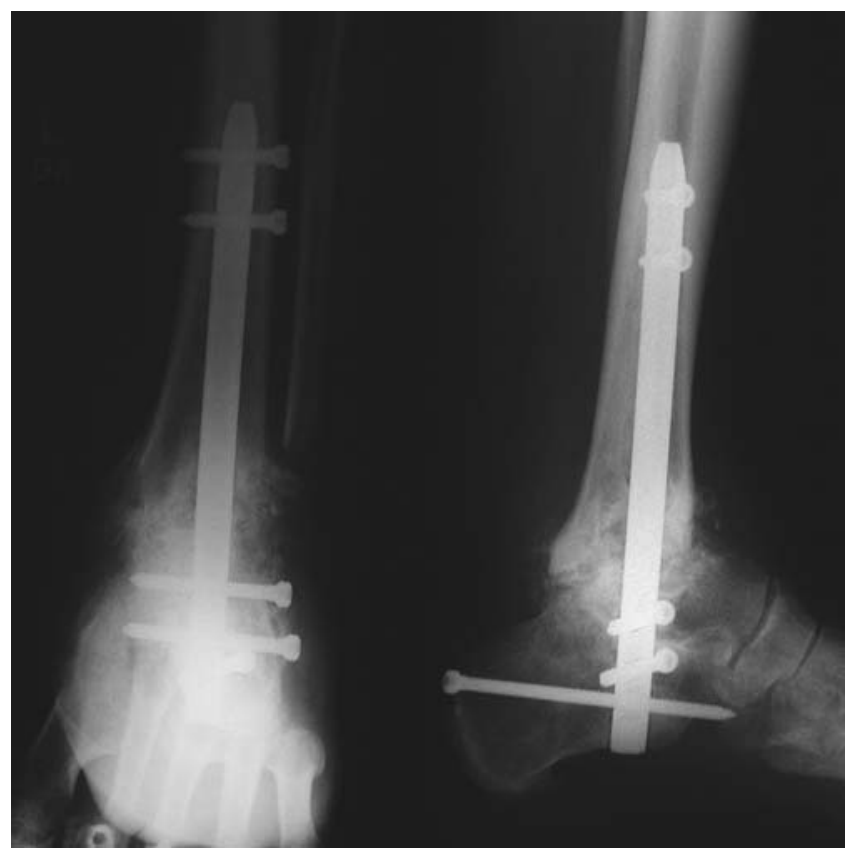

Fig. 5b

Radiographs showing a) a non-infected and loose Buechel-Pappas total ankle replacement and b) a hindfoot fusion using a locked retrograde intramedullary nail.

\section{Discussion}

Surgery for a failed TAR is directed towards restoring alignment, length, stability and function of the limb. Secondgeneration prostheses have made TAR a viable alternative to ankle arthrodesis in the management of degenerative disease of the ankle. ${ }^{7,10,12,13,16}$ The failed and infected ankle replacement, however, remains a surgical challenge, experience of which is limited.

The technical considerations of primary TAR, particularly optimal positioning of the implant and the specific complications, have been described by several authors. ${ }^{15,23,24}$ The management of aseptic loosening can be divided into that in which the joint replacement can be salvaged and that in which the ankle must be fused. ${ }^{15}$ Factors which must be taken into account include loss of bone stock in the tibia and/or talus, fracture or erosion of either malleolus and compromise of the soft-tissues. In addition, significant bone defects may require reconstruction with an autograft or an allograft, each with their own complications. Other considerations include the potential for wound dehiscence after revision surgery, the presence of avascular necrosis of the talar dome and subtalar osteoarthritis. ${ }^{14,15,25-28}$ The armamentarium of revision prostheses is much less than that for other large joints.

The surgical options include a revision TAR, salvage fusion or amputation. The choice of procedure depends on the cause of failure, the surgeon's expertise and experience and an informed discussion with the patient. Revision is contraindicated in the presence of active infection and poor vascular supply, and inadvisable in the presence of large bony defects which increase the chances of malalignment and instability of the prosthesis with resultant early failure. ${ }^{14,15,25-28}$ A revision TAR has the advantage of preserving movement at the ankle, but has a higher rate of complications and an increased risk of early failure compared with a primary TAR. While ankle fusion will limit movement and shorten the limb, it is often preferred since it is currently more reliable at providing relief from pain and gives a stable platform on which to bear weight. There are complications such as nonunion which may require further revision if pain or instability are predominant features. ${ }^{29}$ The difficulties in achieving fusion after a failed TAR have been addressed in several studies ${ }^{25,28,30}$ and Kitaoka and Romness $^{26}$ and Kitaoka ${ }^{27}$ reported using external fixation to fuse a nonunion. The difficulty appears to be with the remaining body of the talus. Fixation fails if the talus is avascular, collapsed or largely absent. The bone loss may necessitate a tibiotalocalcaneal fusion. With the availability of intramedullary devices, hindfoot fusion is often preferable. Amputation is rarely a first-line treatment, but should always remain an option especially in the presence of active infection, inadequate vascular supply and poor soft-tissue cover. An early amputation may provide better long-term function and decrease the need for multiple procedures aimed at salvage of the limb. Several studies have reported secondary procedures after TAR; Spirt et $\mathrm{al}^{16}$ noted a high rate of re-operation after TAR. In their study of 306 patients undergoing a primary TAR, $85(28 \%)$ underwent a 
re-operation. The most common reason was the formation of heterotopic bone and component malalignment. Valderrabano et $\mathrm{al}^{10}$ reported a revision rate of $21 \%$ in 65 patients who had undergone a STAR procedure. Schernberg 6 reporting on a series of 131 TARs, followed up for between one and seven years, had a revision rate of almost 10\% (13 patients) and, similarly, Hintermann ${ }^{12}$ revised seven of 50 TARs $(14 \%)$.

The management of failed TARs has not been extensively reported in the literature. Zwipp and Grass ${ }^{18}$ reported four patients undergoing ankle arthrodesis after a failed TAR. Two achieved a painless gait. In one patient, the arthrodesis had not achieved union by nine months. Fusion was achieved by a revision procedure. More recently, Johl et $\mathrm{al}^{17}$ presented a case report in which a retrograde short femoral nail was used to achieve fusion after a hindfoot arthrodesis for a failed TAR. Groth and Fitch ${ }^{25}$ advised direct tibiotalar fusion without grafting for a septic arthroplasty, but such an approach may lead to unacceptable shortening. Bishop, Wood and Sheetz ${ }^{31}$ studied the use of a free vascularised bone graft to the septic ankle using either the contralateral fibula or an iliac musculocutaneous graft, but reported reactivation of infection in $50 \%$ of patients. Thordarson et $\mathrm{al}^{32}$ reported better results with a more aggressive debridement and a delay in bone grafting. Aggressive debridement is the key to eliminating infection after joint replacement. Only after the bone is free from any type of infection can definitive treatment be planned and consistent results be produced.

Our series of 16 patients with a failed ankle replacement undergoing revision surgery is the largest to date. An algorithm for the treatment of the failed ankle replacement has been developed and implemented in our unit (Fig. 1). Patients were allocated to one of two methods of treatment depending on the presence of infection. Those with septic loosening were treated by either a hindfoot fusion or a revision TAR. The latter was considered to be the method of choice. Of the 14 patients identified with aseptic loosening, only five underwent a revision TAR. The remaining patients were considered to be unsuitable for revision. Seven were considered to have inadequate bone stock. In five of these, revision of one or both components of their primary replacement had already been performed. A further two patients had undergone a heel-shift procedure to correct hindfoot alignment.

Overall, excluding our patient who underwent amputation, the patients undergoing hindfoot fusion (8 of 10) reported less pain after the procedure compared with those undergoing revision (3 of 5). While the remaining two patients who had revision TAR were satisfied with their post-operative range of ankle movement and function, the persistent pain remained a concern. One of these patients already had radiological signs of impending failure.

Infection was considered to be a contraindication to revision TAR or fusion in the two patients with septic loosening. One of these underwent a primary amputation because of ongoing infection and vascular compromise. The other elected for limb salvage with fusion being achieved using an Ilizarov frame at nine months after revision. However, fusion with an Ilizarov frame exposes the patient to a protracted course of treatment and the complications which are ubiquitous to fine-wire frames. This approach can only be recommended in cases in which it is thought that the limb can be salvaged or in which leg lengthening is required.

We recommend a two-stage revision as being mandatory in managing the infected TAR. A multidisciplinary approach is also of importance and we are fortunate to have a dedicated bone infection unit onsite. Revision surgery should not be performed in the presence of unresolved infection and surgeons should be guided as to the safe time for re-implantation by physicians experienced in managing failed and infected joint replacements. In our series, patients who had previously undergone a revision procedure did less well after definitive management. A followup of 12 months is insufficient to make any long-term predictions but we are of the opinion that rather than subjecting the patient to multiple small operations, a single definitive revision procedure may be more appropriate.

The decision to revise or fuse is a difficult choice for the surgeon and the patient. Many factors need to be taken into account as outlined above. Currently, hindfoot fusion appears to be preferable in non-infected cases, but with limited follow-up, definite conclusions cannot be drawn. With experience of only two infected cases, management in this scenario remains uncertain, but both amputation and fusion with an external fixator should be considered, each with its own advantages and disadvantages. In addition, in the future determination of pre- and post-operative outcome scores will allow a direct comparison of the clinical results between the methods of treatment. Our results confirm that revision of a failed TAR is a difficult procedure with unreliable results. Such surgery should be performed by surgeons experienced in primary TAR and ideally in units where multidisciplinary support is available.

No benefits in any form have been received or will be received from a commercial party related directly or indirectly to the subject of this article.

\section{References}

1. Bolton-Maggs BG, Sudlow RA, Freeman MA. Total ankle arthroplasty: a longterm review of the London Hospital experience. J Bone Joint Surg [Br] 1985;67-B: 785-90.

2. Helm R, Stevens J. Long-term results of total ankle replacement. J Arthroplasty 1986;1:271-7.

3. Buechel FF, Pappas MJ. Survivorship and clinical evaluation of cementless, meniscal-bearing total ankle replacements. Semin Arthroplasty 1992;3:43-50.

4. Wynn AH, Wilde AH. Long-term follow-up of the Conaxial (Beck-Steffee) total ankle arthroplasty. Foot Ankle 1992;13:303-6.

5. Pyevich MT, Saltzman CL, Callaghan JJ, Alvine FG. Total ankle arthroplasty: a unique design: two to twelve-year follow-up. J Bone Joint Surg [Am]1998;80-A:1410-20.

6. Schernberg F. Current results of ankle arthroplasty: European multi-center study of cementless ankle arthroplasty. In: Kofoed $\mathrm{H}$, ed. Current status of ankle arthroplasty. Berlin: Springer, 1998:41-6.

7. Anderson T, Montgomery F, Carlsson A. Uncemented STAR total ankle prostheses: three to eight-year follow-up of fifty-one consecutive ankles. J Bone Joint Surg [Am] 2003:85-A:1321-9 
8. Wood PL, Deakin S. Total ankle replacement: the results in 200 ankles. J Bone Joint Surg [Br] 2003;85-B:334-41.

9. Buechel FF Sr, Buechel FF Jr, Pappas MJ. Ten-year evaluation of cementless Buechel-Pappas meniscal bearing total ankle replacement. Foot Ankle Int 2003;24: 462-72.

10. Valderrabano V, Hintermann B, Dick W. Scandinavian total ankle replacement: 3.7-year average follow-up of 65 patients. Clin Orthop 2004;424:47-56.

11. Kitaoka HB, Patzer GL. Clinical results of the Mayo total ankle arthroplasty. J Bone Joint Surg [Am] 1996;78-A:1658-64.

12. Hintermann B. Short- and mid-term results with the STAR total ankle prosthesis. Orthopade 1999;28:792-803.

13. Wood PL. Experience with the STAR ankle arthroplasty at Wrightington Hospital, UK. Foot Ankle Clin 2002;7:755-64.

14. Wapner KL. Salvage of failed and infected total ankle replacements with fusion. Instr Course Lect 2002;51:153-7.

15. Stamatis ED, Myerson MS. How to avoid specific complications of total ankle replacement. Foot Ankle Clin 2002;7:765-89.

16. Spirt AA, Assal M, Hansen ST Jr. Complications and failure after total ankle arthroplasty. J Bone Joint Surg [Am]2004;86-A:1172-8.

17. JohI C, Kircher J, PohImann K, Jansson V. Management of failed total ankle replacement with a retrograde short femoral nail: a case report. J Orthop Trauma 2006;20:60-5.

18. Zwipp H, Grass R. Ankle arthrodesis after failed joint replacement. Oper Orthop Traumato/ 2005;17:518-33.

19. Myerson MS, Miller SD. Salvage after complications of total ankle arthroplasty. Foot Ankle Clin 2002;7:191-206.

20. Berendt AR, McLardy-Smith P. Prosthetic joint infection. Curr Infect Dis Rep 1999; 1:267-72.
21. Pandey R, Berendt AR, Athanasou NA. Histological and microbiological findings in non-infected and infected revision arthroplasty tissues: the OSIRIS Collaborative Study Group, Oxford Skeletal Infection Research and Intervention Service. Arch Orthop Trauma Surg 2000;120:570-4.

22. Atkins BL, Athanasou N, Deeks JJ, et al. Prospective evaluation of criteria for microbiological diagnosis of prosthetic-joint infection at revision arthroplasty: the OSIRIS Collaborative Study Group. J Clin Microbio/ 1998;36:2932-9.

23. Saltzman CI, Alvine FG. The Agility total ankle replacement. Instr Course Lect2002 51:129-33.

24. Conti SF, Wong YS. Complications of total ankle replacement. Clin Orthop 2001;391 105-14.

25. Groth HE, Fitch HF. Salvage procedures for complications of total ankle arthroplasty. Clin Orthop 1987;224:244-50.

26. Kitaoka HB, Romness DW. Arthrodesis for failed ankle arthroplasty. J Arthroplasty 1992;7:277-84.

27. Kitaoka HB. Fusion techniques for failed total ankle arthroplasty. Semin Arthroplasty 1992:3:51-7.

28. Stauffer RN. Salvage of painful total ankle arthroplasty. Clin Orthop 1982;170:184-8

29. Kitaoka HB. Salvage of nonunion following ankle arthrodesis for failed total ankle arthroplasty. Clin Orthop 1991;268:37-43.

30. Carlsson AS, Montgomery F, Besjakov J. Arthrodesis of the ankle secondary to replacement. Foot Ankle Int 1998;19:240-5.

31. Bishop AT, Wood MB, Sheetz KK. Arthrodesis of the ankle with a free vascularised autogenous bone graft: reconstruction of segmental loss of bone secondary to osteomyelitis, tumor, or trauma. J Bone Joint Surg [Am] 1995;77-A:1867-75.

32. Thordarson DB, Ahlmann E, Shepherd LE, Patzakis MJ. Sepsis and osteomyelitis about the ankle joint. Foot Ankle Clin 2000;5:913-28. 